\title{
Distinctive profiles of small RNA couple inverted repeat-induced post-transcriptional gene silencing with endogenous RNA silencing pathways in Arabidopsis
}

\author{
TADEUSZ WROBLEWSKI, ${ }^{1}$ MARTA MATVIENKO ${ }^{1,5}$ URSZULA PISKUREWICZ, ${ }^{1}$ HUAQIN XU, ${ }^{1}$ \\ BELINDA MARTINEAU, ${ }^{1}$ JOAN WONG, ${ }^{1}$ MANJULA GOVINDARAJULU, ${ }^{1}$ ALEXANDER KOZIK, ${ }^{1}$ \\ and RICHARD W. MICHELMORE ${ }^{1,2,3,4}$ \\ ${ }^{1}$ The Genome Center, University of California, Davis, Davis, California 95616, USA \\ ${ }^{2}$ Department of Plant Science, ${ }^{3}$ Department of Molecular and Cellular Biology, ${ }^{4}$ Department of Medical Microbiology and Immunology, \\ University of California, Davis, Davis, California 95616, USA
}

\begin{abstract}
The experimental induction of RNA silencing in plants often involves expression of transgenes encoding inverted repeat (IR) sequences to produce abundant dsRNAs that are processed into small RNAs (sRNAs). These sRNAs are key mediators of posttranscriptional gene silencing (PTGS) and determine its specificity. Despite its application in agriculture and broad utility in plant research, the mechanism of IR-PTGS is incompletely understood. We generated four sets of 60 Arabidopsis plants, each containing IR transgenes expressing different configurations of uidA and CHALCONE SYNTHASE (At-CHS) gene fragments. Levels of PTGS were found to depend on the orientation and position of the fragment in the IR construct. Deep sequencing and mapping of sRNAs to corresponding transgene-derived and endogenous transcripts identified distinctive patterns of differential sRNA accumulation that revealed similarities among sRNAs associated with IR-PTGS and endogenous sRNAs linked to uncapped mRNA decay. Detailed analyses of poly-A cleavage products from At-CHS mRNA confirmed this hypothesis. We also found unexpected associations between sRNA accumulation and the presence of predicted open reading frames in the trigger sequence. In addition, strong IR-PTGS affected the prevalence of endogenous sRNAs, which has implications for the use of PTGS for experimental or applied purposes.
\end{abstract}

Keywords: heterochromatic siRNA; transitivity; RNA decay; hairpin RNA; chalcone synthase; translation

\section{INTRODUCTION}

Small RNAs (sRNA) in plants exhibit a range of size classes, predominantly from 20 to $24 \mathrm{nt}$, that are characteristic of their origin and the mechanism by which they are generated. Depending on their origin and structure, dsRNA triggers of RNAi are cleaved by one or more Dicer-like nucleases (DCLs) (Zamore et al. 2000; Bernstein et al. 2001); four DCLs are known in Arabidopsis (Henderson et al. 2006). DCL4 and DCL2 can process perfectly complementary dsRNA to produce 20- to 22-nt long sRNAs referred to as short-interfering RNAs (siRNAs) (Hamilton and Baulcombe 1999; Hamilton et al. 2002; Hannon 2002; Tang et al. 2003; Bouche et al. 2006). DCL1 cleaves partially paired dsRNA precursors to produce 21-nt long sRNAs known as microRNAs (miRNAs) (Denli and Hannon 2003; Bartel 2004)

\footnotetext{
${ }^{5}$ Present address: CLC bio, a QIAGEN Company, Davis, CA 95616, USA Corresponding author: tadeusz.academicus@gmail.com

Article published online ahead of print. Article and publication date are at http://www.rnajournal.org/cgi/doi/10.1261/rna.046532.114. Freely available online through the RNA Open Access option.
}

and DCL3 is involved in biogenesis of 24-nt long repeat-associated sRNAs known as heterochromatic siRNAs (hcsiRNAs) (Xie et al. 2005). When associated with one of the ARGONAUTE proteins (AGO) in an RNA-induced silencing complex (RISC), 20- to 21-nt long siRNAs and miRNAs direct sequence-specific cleavage of complementary target RNAs or interfere with their translation (Hammond et al. 2000; Hannon 2002; Brodersen et al. 2008). Twenty-one nucleotides long siRNAs may also participate in siRNA-dependent methylation of genomic loci (Pontier et al. 2012), a function traditionally attributed to the 24-nt long species and linked to chromatin modifications and transcriptional silencing (Hamilton et al. 2002; Zilberman et al. 2003; Liu et al. 2004; Law and Jacobsen 2010).

Endogenous dsRNA precursors of sRNA may be produced in eukaryotic cells through the transcription of selfcomplementary sequences and/or through the activity of

(C) 2014 Wroblewski et al. This article, published in $R N A$, is available under a Creative Commons License (Attribution 4.0 International), as described at http://creativecommons.org/licenses/by/4.0/. 
RNA-dependent RNA polymerases (RDRs) that are capable of synthesizing complementary strands using preexisting single-stranded templates (Dalmay et al. 2000; Mourrain et al. 2000; Curaba and Chen 2008; Garcia et al. 2012). RDRs are also essential for the amplification of RNA silencing that frequently follows initial RISC-mediated cleavage of the target RNA and can result in the production of secondary sRNA. One such type of secondary sRNAs, known as transitive sRNAs, is produced from the sequences linked to the primary cleavage target during RNA silencing (Voinnet et al. 1998; Vaistij et al. 2002; Voinnet 2008). As with DCLs, particular RDRs are associated with the different pathways of sRNA biogenesis; for example, RDR2, along with DCL3, is critical for hc-siRNA production (Xie et al. 2004; Lu et al. 2006), and RDR6 generates perfectly complementary dsRNA substrates for DCL4 during biogenesis of transitive sRNA and trans-acting siRNA (ta-siRNA) (Peragine et al. 2004; Vazquez et al. 2004; Allen et al. 2005; Brodersen and Voinnet 2006). In Arabidopsis, amplification of RNA silencing requires not only RDR6 but also a helicase activity, which can be provided by enzymes like Silencing Defective 3 (SDE3) (Dalmay et al. 2001), a model for how enzymes may facilitate reuse of an original template and thereby accelerate amplification, as previously proposed (Garcia et al. 2012).

The various DCLs process dsRNAs that may occur in a cell as a consequence of viral infection or transgene expression (Chuang and Meyerowitz 2000; Llave et al. 2002). In these cases the trigger and the target are represented by the same sequence (trigger/target), but the sRNA products can also affect other RNAs present in the cell that share sufficient sequence identity with the trigger. Abundant dsRNA known as hairpin RNA (hpRNA) can be produced in plant cells through the expression of transgenes containing inverted repeats (IR) of the trigger sequence. This technique, referred to as IR post-transcriptional gene silencing (IR-PTGS), is commonly used to down-regulate endogenous mRNAs (Smith et al. 2000). Separation of inverted repeats by a functional intron sequence enhances the efficiency of PTGS; dsRNA produced from these transgenes is referred to as intron-spliced hairpin RNA (ihpRNA) (Smith et al. 2000). Although IR-PTGS has agricultural applications and is frequently used as a research tool, the mechanisms involved in ihpRNA processing are not well understood (Brodersen and Voinnet 2006; Brosnan and Voinnet 2011). Similarities between ihpRNA and the endogenous precursors of ta-siRNA (perfectly complementary dsRNA) as well as the abundant 21-nt long sRNAs associated with IR-PTGS, suggests the involvement of DCL4 (Gasciolli et al. 2005; Xie et al. 2005). In the absence of DCL4, DCL2 can process long, perfectly complementary dsRNAs that comprise endogenous DCL4 substrates to produce 22-nt long sRNAs (Gasciolli et al. 2005; Xie et al. 2005; Brodersen and Voinnet 2006). Forward genetic screens imply that short-distance signaling during IR-PTGS requires components of the endogenous 24-nt siRNA pathway (CLASSY1 [CLSY1], NUCLEAR RNA POLYMERASE D1a
[NRPD1a], and RDR2) but not the components of the endogenous, RDR6-dependent, 21-nt siRNA pathway (Dunoyer et al. 2005; Smith et al. 2007; Brosnan and Voinnet 2011). Long-distance signaling involving vascular tissue requires components of both pathways (Brosnan et al. 2007; Dunoyer and Voinnet 2008; Jauvion et al. 2010).

Here we present a detailed analysis of sRNAs associated with IR-PTGS in Arabidopsis. We sequenced seven sRNA libraries produced from large numbers of transgenic plants expressing four different configurations of chimeric IR constructs designed to trigger RNA silencing of two different genes. Mapping of sRNA sequences to their corresponding transgene-derived and endogenous transcripts revealed distinctive patterns of sRNA accumulation. Analyses of these patterns linked endogenous pathways of RNA silencing with IR-PTGS and identified an unexpected correlation between the presence of ORFs in RNA sequences and sRNA accumulation. We also found that high levels of IR-PTGS affected endogenous levels of sRNAs, primarily by decreasing hc-siRNAs.

\section{RESULTS}

\section{Configuration of the ihpRNA trigger sequence affects efficiency of IR-PTGS in Arabidopsis}

To investigate the effects of ihpRNA configuration on the efficiency of IR-PTGS, we produced four sets of Arabidopsis transgenics (ecotype Ws-0), each transformed with one of four constructs designed to express ihpRNAs in different configurations. Each construct contained a 342-bp fragment of the bacterial uidA gene (Jefferson et al. 1987) fused to a 372-bp fragment of Arabidopsis CHALCONE SYNTHASE (At-CHS) in the sense (coding) and antisense orientations (Fig. 1A; Supplemental Fig. S1). Fifty-nine or 60 independent transgenic individuals were generated for each construct. All $\mathrm{T}_{1}$ plants were evaluated for their ability to silence uidA expression based on the level of transient ectopic expression of $\beta$-glucuronidase (GUS) (Fig. 1B). The $\mathrm{T}_{2}$ progenies of these plants were assayed for silencing of At-CHS, based on their ability to produce anthocyanin under stressful conditions (Fig. 1C).

Silencing of GUS in $\mathrm{T}_{1}$ plants was strongly correlated with silencing of At-CHS in the corresponding $\mathrm{T}_{2}$ progenies ( $r=0.83 ; P \geq 0.01)$; however, the efficiency of silencing each gene was influenced by the configuration of the trigger sequence in the ihpRNA construct. Silencing of GUS in $\mathrm{T}_{1}$ plants was less effective $(P \leq 0.1)$ when the uidA trigger sequence was close to the $5^{\prime}$ end of the ihpRNA and in the sense (coding) orientation (gus ${ }^{\mathrm{S}}$-chs ${ }^{\mathrm{S}} \wedge$ chs $^{\mathrm{A}}{ }^{\text {-gus }}{ }^{\mathrm{A}}$; ${ }^{\mathrm{S}}$ and ${ }^{\mathrm{A}}=$ sense and antisense orientation of the fragment, respectively, $\wedge=$ the position of the intron) (Supplemental Fig. S1) as compared with plants transformed with the other three constructs. Similarly, silencing of At-CHS was significantly 

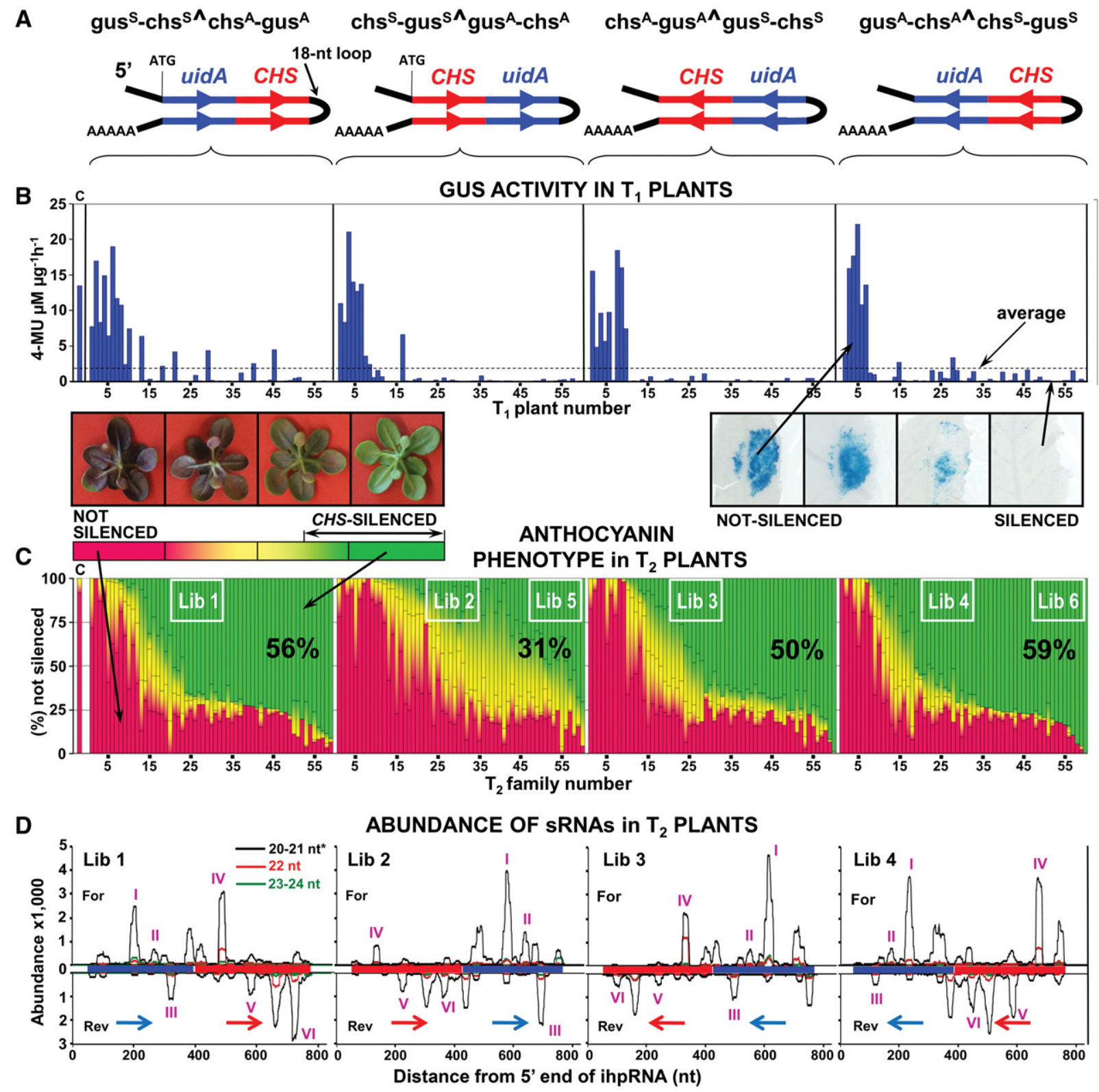

FIGURE 1. Phenotypes and sRNA profiles in transgenic Arabidopsis ecotype Ws- 0 transformed with four constructs configured to produce different ihpRNAs. (A) Predicted ihpRNA structures produced by expression from the integrated T-DNA. (B) Expression of $\beta$-glucuronidase in the leaves of transgenic $\mathrm{T}_{1}$ plants measured after Agrobacterium-mediated transient expression of the uidA (GUS) gene. Each bar shows the average GUS activity per $\mathrm{T}_{1}$ plant. $(C)$ Percentage of $\mathrm{T}_{2}$ plants derived from each $\mathrm{T}_{1}$ plant shown in $(B)$ sorted into four classes depending on anthocyanin production: (red) not silenced, (red-yellow) weakly silenced, (yellow-green) moderately silenced, and (green) strongly silenced. The white rectangle indicates the plants used for construction of sRNA and RNA-Seq libraries. (c) control wild-type plants, Arabidopsis thaliana ecotype Ws-0. Each bar corresponds to 48 or more $\mathrm{T}_{2}$ individuals derived from each $\mathrm{T}_{1}$ plant shown in $(B) .(D)$ Abundance of transgene-derived sRNA sequences corresponding to the ihpRNA sequences. Roman numerals (purple) indicate peaks of sRNA accumulation compared between libraries. Abundance of sRNA in 1 million reads per library is shown. $\left({ }^{*}\right) 20$-nt long sRNA accounted for up to $2 \%$ of the 20 - to 21 -nt ihpRNA-derived sRNA shown in the alignment.

less effective $(P \leq 0.05)$ when the corresponding sequence was located closer to the $5^{\prime}$ end of the ihpRNA ( $\mathrm{chs}^{\mathrm{S}}$ gus $^{\mathrm{S}} \wedge$ gus $^{\mathrm{A}}-$ chs $^{\mathrm{A}}$ ) in the sense (coding) orientation, as compared with the other three constructs. Thus, both the presence of the ORF and the position of the sequence in the ihpRNA affected efficiency of IR-PTGS.

\section{Configuration of ihpRNA trigger affects sRNA accumulation}

To determine whether the observed differences in silencing were associated with differences in sRNA accumulation, we performed deep sequencing of seven sRNA libraries. For 
each construct, sRNAs were extracted from a pool of $120 \mathrm{~T}_{2}$ plants that exhibited intermediate (Lib 1-4) and strong levels of silencing (Lib 5 and Lib 6) (Fig. 1C). The sRNAs extracted from wild-type, nontransgenic plants were used for the reference library (Lib 0). Approximately 6 million sRNA sequences were obtained from each library using Illumina sequencing. The ihpRNA-derived sRNAs represented between $5.7 \%$ and $9.6 \%$ of the total sRNA in each library; most $(80.0 \%-87.9 \%)$ were 20 - to 21 -mers, consistent with previous reports of involvement of this size class of sRNA in dsRNA-mediated silencing (Hamilton et al. 2002; Llave et al. 2002; Dunoyer et al. 2005). Two other abundant classes of transgene-derived sRNAs were 22-mers accounting for $7.9 \%-13.6 \%$ and 23 - to 24 -mers accounting for $1.8 \%-$ $5.2 \%$ of all sRNAs in each library.

BLAST analysis and mapping of the sRNAs to the corresponding ihpRNA sequences revealed peaks of sRNA accumulation in both uidA and At-CHS sequences, rather than an even or random distribution across the length of the trigger sequences (Fig. 1D). The positions of these peaks with respect to each trigger sequence were the same for all four ihpRNAs, indicating some form of dependence on the sequence (see below) rather than the configuration of the ihpRNA construct (Fig. 1D). However, the abundance of sRNAs associated with each peak varied substantially depending on the position and the orientation of the trigger sequence within the construct (Fig. 1A,D). Significantly fewer sRNAs were produced when the fragment of At-CHS or uidA was adjacent to the $5^{\prime}$ end of the ihpRNA in the sense (coding) orientation, compared with the antisense orientation $(P \leq 0.01)$. When the trigger fragment was adjacent to the intron/loop, sRNAs were produced at higher levels regardless of sequence orientation. Accordingly, the abundance of sRNAs associated with each peak was inversely correlated ( $r=-0.81 ; P \leq 0.01)$ with the sequence's distance from the loop/intron (Fig. 2). These construct-dependent differences

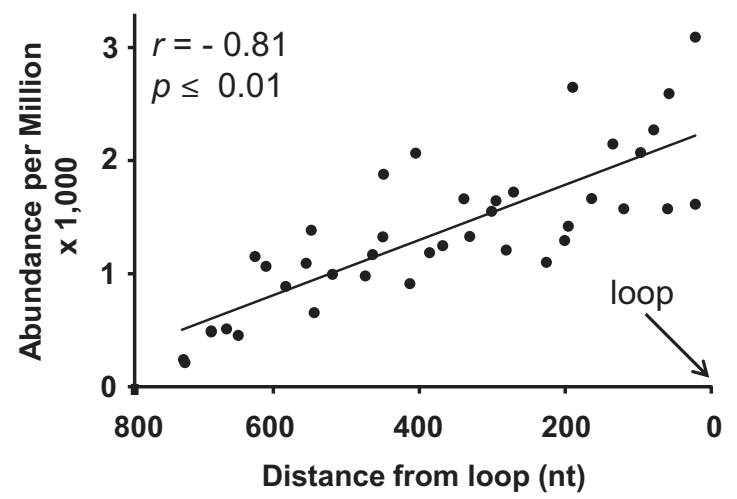

FIGURE 2. Correlation between the abundance of transcripts in ten major peaks of sRNA production and the position of these peaks in the ihpRNA. The five largest peaks corresponding to the At-CHS and uidA (GUS) fragments were selected and the abundance was calculated for all four sRNA libraries (Libs 1-4). ( $r$ ) correlation coefficient. in sRNA accumulation paralleled the phenotypic differences in GUS and At-CHS silencing; higher sRNA accumulation was correlated with stronger silencing. From this experiment we conclude that levels of sRNA production and IR-PTGS efficiency are highest when the trigger sequence is closer to the loop in ihpRNA. In addition, the presence of an ORF in the first ( $5^{\prime}$ most) arm of IR decreases sRNA production and efficiency of IR-PTGS.

\section{ihpRNA-derived sRNAs and sRNAs linked to uncapped mRNA decay are similar, and their accumulation is associated with the presence of predicted ORFs in the trigger sequence}

To investigate whether ihpRNA-derived sRNAs in our experiments were similar to those produced endogenously, we searched the control library (Lib 0) generated from wild-type ecotype Ws-0 plants for At-CHS-derived sRNAs. Among over 6 million reads in this library we found only one 21-mer corresponding to a region of the At-CHS mRNA upstream of the trigger fragment used in our ihpRNA constructs. In contrast, we retrieved abundant 20to 21-nt long At-CHS-derived sRNAs from the publicly available sRNA database for Arabidopsis ecotype Col-0 (http:/ mpss.udel.edu/at_sbs/). Interestingly, these sRNAs in ecotype Col-0 were very similar to the highly abundant sRNA species corresponding to the At-CHS trigger fragment in our transgenic Ws-0 plants; i.e., both sets of sRNAs mapped to the same peaks in the At-CHS sequence that occurred with an approximate periodicity of 79 nt (Fig. 3A,B).

Over $65 \%$ of the At-CHS-derived sRNAs from Col-0 in the database originated from a library made from the ein56/abh11 mutant, which is defective in mRNA capping and deficient in the exoribonuclease 4 (XRN4) responsible for $5^{\prime} \rightarrow 3^{\prime}$ degradation of uncapped mRNA (Gregory et al. 2009). The ein56/abh11 mutations caused elevated sRNA production and mRNA degradation for 133 distinct genes, one of which is At-CHS (Gregory et al. 2009). Interestingly, sRNAs from the ein56/abh11 mutant precisely (within a few nucleotides) matched the fragment of the At-CHS mRNA corresponding to reading frame 1 and to a secondary predicted ORF in reading frame 2 that extends past the first ORF (Fig. 3B). To investigate this phenomenon further, we analyzed all of the 133 genes affected by RNAi-mediated decay in this ein56/abh11 mutant (Gregory et al. 2009). For 90 of these genes, accumulation of sRNA was primarily associated with predicted primary or secondary ORF sequences (Supplemental Fig. S2). For the remaining 43 genes, the representation of corresponding sRNAs was too low for analysis. Therefore, ihpRNA-derived sRNAs in our transgenic plants are similar to the endogenous sRNAs associated with mRNA decay, and the accumulation of the latter appears to be influenced by the presence of ORFs in the target RNA. 

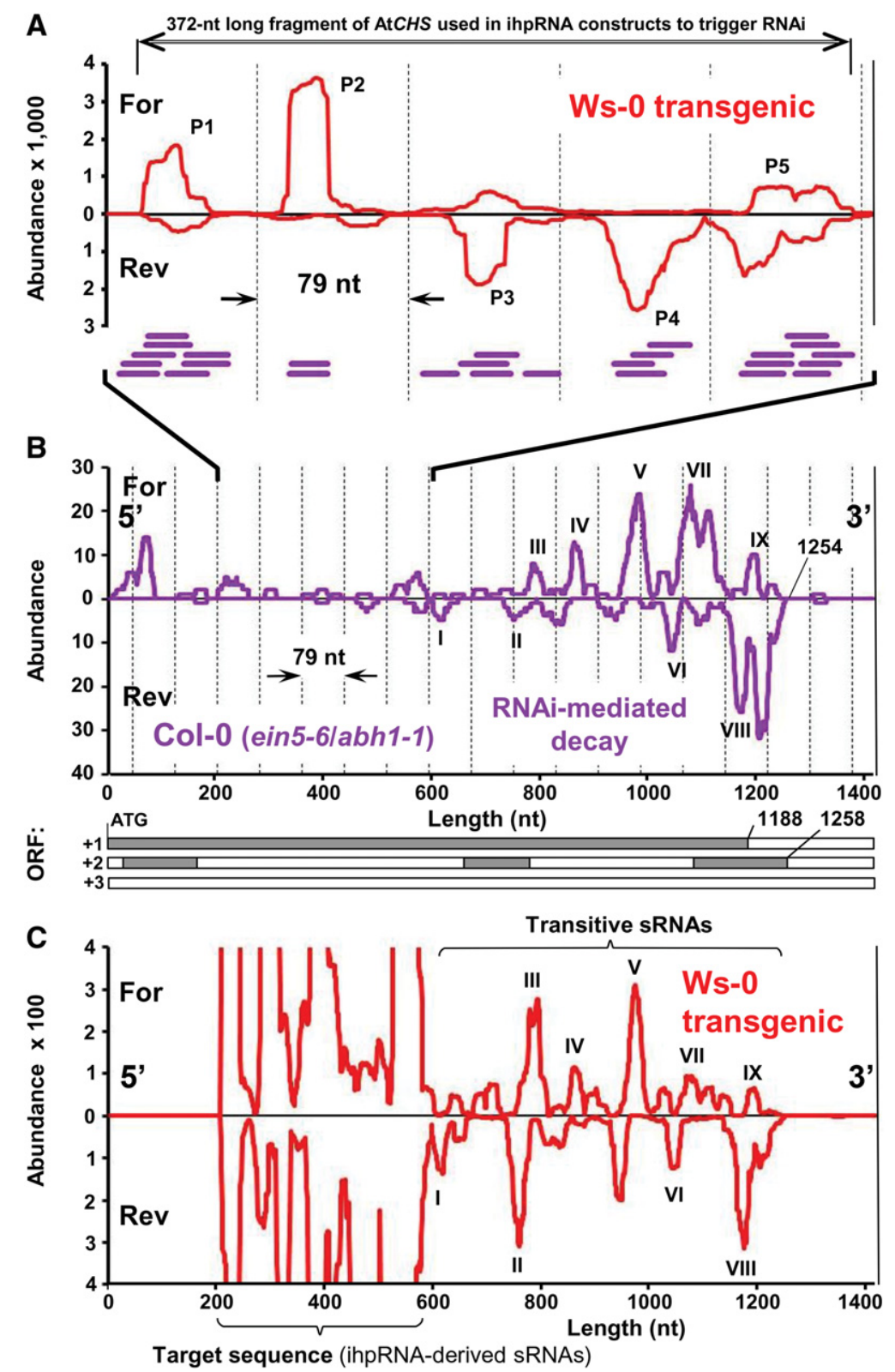

FIGURE 3. Analysis of endogenous, transgene-derived and transitive sRNAs corresponding to At-CHS* in Arabidopsis. (A) Similarity in the distribution of ihpRNA-derived sRNAs (red) in transgenic Ws-0 plants and sRNA associated with uncapped mRNA decay in the ein5-6/abh1-1 mutant in Col-0 ecotype background (purple bars). Sequences of sRNA were mapped to the fragment of At-CHS* sequence used in the ihpRNA construct. (B) Abundance of nonredundant sRNA sequences corresponding to the full-length At-CHS* identified in Col-0 (ein5-6/abh1-1 mutant) (Gregory et al. 2009). sRNAs match the $3^{\prime}$ fragment of the At-CHS* mRNA that corresponds to the primary and secondary ORFs in frame 1 and 2, respectively. Roman numerals indicate peaks of sRNA accumulation for comparison to transitive sRNAs shown in $(C)$. $(C)$ Representation of sRNA transcripts corresponding to the full-length At-CHS* in transgenic Ws-0 plants showing numerous transitive sRNAs matching the region downstream from $\left(3^{\prime}\right)$ but not upstream of $\left(5^{\prime}\right)$ the RNAi target sequence. Abundance corresponds to representation per nucleotide per million reads. Since the abundance of AtCHS mRNA-derived sRNA did not exceed 300 per million per $n t$ and the abundance of reads corresponding to the trigger/target fragment approached 3500 per million per nucleotide, we assumed that sRNAs corresponding to the trigger/target fragment originated from ihpRNA processing. *mRNA sequences of At-CHS in ecotypes Col-0 and Ws-0 are identical.

\section{Patterns of $3^{\prime}$ transitive sRNA production match patterns of sRNA accumulation associated with uncapped mRNA decay}

Induction of RNA silencing has been reported to trigger production of secondary, transitive sRNAs from the regions flanking the target sequence (Voinnet et al. 1998; Vaistij et al. 2002; Moissiard et al. 2007; Voinnet 2008). We searched our sRNA libraries for secondary sRNAs corresponding to regions of the fulllength At-CHS mRNA outside of the trigger/target sequence used in our ihpRNA constructs. In the libraries made from transgenic plants (Libs 1-4), we found numerous sRNAs matching the region downstream, but not upstream of the trigger/target sequence (Fig. 3C). Interestingly, the pattern of these transitive sRNAs corresponded exactly to the pattern of sRNAs derived from native AtCHS in the ecotype Col-0 ein5-6/abh1-1 mutant described above (Fig. 3B,C). Therefore we concluded that the mechanism of sRNA biogenesis may be the same in both experimental systems: (1) RNAi-mediated degradation of uncapped mRNA in the absence of a $5^{\prime} \rightarrow 3^{\prime}$ exoribonuclease in the ein5/abh1-1 mutant and (2) $3^{\prime}$ transitivity in our Ws-0 transgenics.

To validate this hypothesis we analyzed At-CHS-derived transcripts in transgenic and wild-type control plants before and after treatment with $5^{\prime}$-phosphate-dependent exonuclease (5'PDE; catalog \# TER51020; www.epibio.com) to degrade uncapped RNA. We constructed 14 RNA-Seq libraries using poly-A RNA purified from five totalRNA preps used to make sRNA libraries (see Materials and Methods). Seven libraries designated ESeq $0-6$ and seven designated RSeq 0-6 were constructed from 5'PDE-treated and untreated polyA RNA, respectively. Libraries ESeq 0-6 and RSeq 0-6 were made from the same total RNA preps as the sRNA libraries $0-6$, respectively (Fig. 1C). Nine million reads from each library were aligned to Arabidopsis rRNA-5S-encoding sequences, 13 reference sequences used previously for transcript normalization (Czechowski et al. 2005), and the 
At-CHS mRNA sequence. Read alignment to the rRNA-5Sencoding sequences revealed a $52 \%$ reduction in mean abundance (reads per kilo base pair per million) from ESeq versus RSeq libraries, consistent with 5'PDE-mediated degradation of residual rRNA in the poly-A RNA samples used for ESeq library construction (Supplemental Table S2). Alignment to 13RS mRNA reference sequences used for normalization showed an average of $16 \%$ increase in abundance $(P \leq 0.05)$ from ESeq versus RSeq libraries (Supplemental Table S2), consistent with 5'PDE-mediated removal of uncapped RNAs during ESeq library construction and a corresponding increase in the relative abundance of species that are insensitive to $5^{\prime} \mathrm{PDE}-$ mediated degradation. Finally, read alignment to the At-CHS mRNA showed that abundance increased by $19 \%$ in ESeq 0 compared with RSeq 0, indicating that At-CHS mRNA in wild-type plants contained the protective $5^{\prime}$ phosphate cap. The insensitivity of At-CHS mRNA to $5^{\prime} \mathrm{PDE}$-mediated degradation in vitro was consistent with its insensitivity to RNAi-mediated degradation in vivo, as indicated by lack of its corresponding sRNA in Lib 0 .

Further evidence for $5^{\prime}$-capped full-length At-CHS mRNA and uncapped products of its cleavage comes from the distribution of RNA-Seq read alignments to the At-CHS sequence. Reads from the wild-type plant libraries (ESeq 0 and RSeq 0) aligned along the entire length of the At-CHS mRNA sequence (Fig. 4A), in contrast to reads from transgenic plant libraries (RSeq 1-6 and ESeq 1-6), for which several trends were observed. First, the increased relative abundance of reads matching the trigger/target fragment in the IR constructs indicates that the ihpRNA transcripts were polyadenylated and hence copurified along with native mRNAs and that they were incompletely processed to produce sRNAs (Fig. 4B,C). As is consistent with the progressive processing of ihpRNA starting from the loop, the distribution of these aligned reads showed clear bias for areas adjacent to the poly-A tail (Fig. 4B,C). Second, the reduction or lack of reads aligning upstream of the trigger/target sequence (Fig. 4B) in contrast to their relative abundance downstream indicates the cleavage of AtCHS mRNA within this region. This bias can be attributed to reduced representation of cleaved $5^{\prime}$ ends in poly-A-selected mRNA during library construction. Third, a relative decrease in reads corresponding to the $3^{\prime}$ AtCHS mRNA fragment in ESeq versus RSeq libraries (Fig. $4 \mathrm{~B}, \mathrm{C}$ ) indicates a deficiency in $5^{\prime}$ phosphate groups that results in sensitivity to $5^{\prime} \mathrm{PDE}$-mediated degradation; this contrasts with an expected increase in coverage as described above for capped full-length At-CHS mRNAs (RSeq 0 versus ESeq 0) (Fig. 4A) and 13RS in all ESeq libraries.

We concluded that in the transgenic plants, ihpRNAderived sRNAs mediate cleavage of the corresponding AtCHS mRNA, generating $3^{\prime}$ uncapped fragments that are directed to the RNAi-mediated decay pathway (Gazzani et al. 2004; Souret et al. 2004; Gregory et al. 2009). In addition, the similarity of sRNA profiles we observed indicates that $3^{\prime}$ transitivity and silencing-mediated RNA decay involve

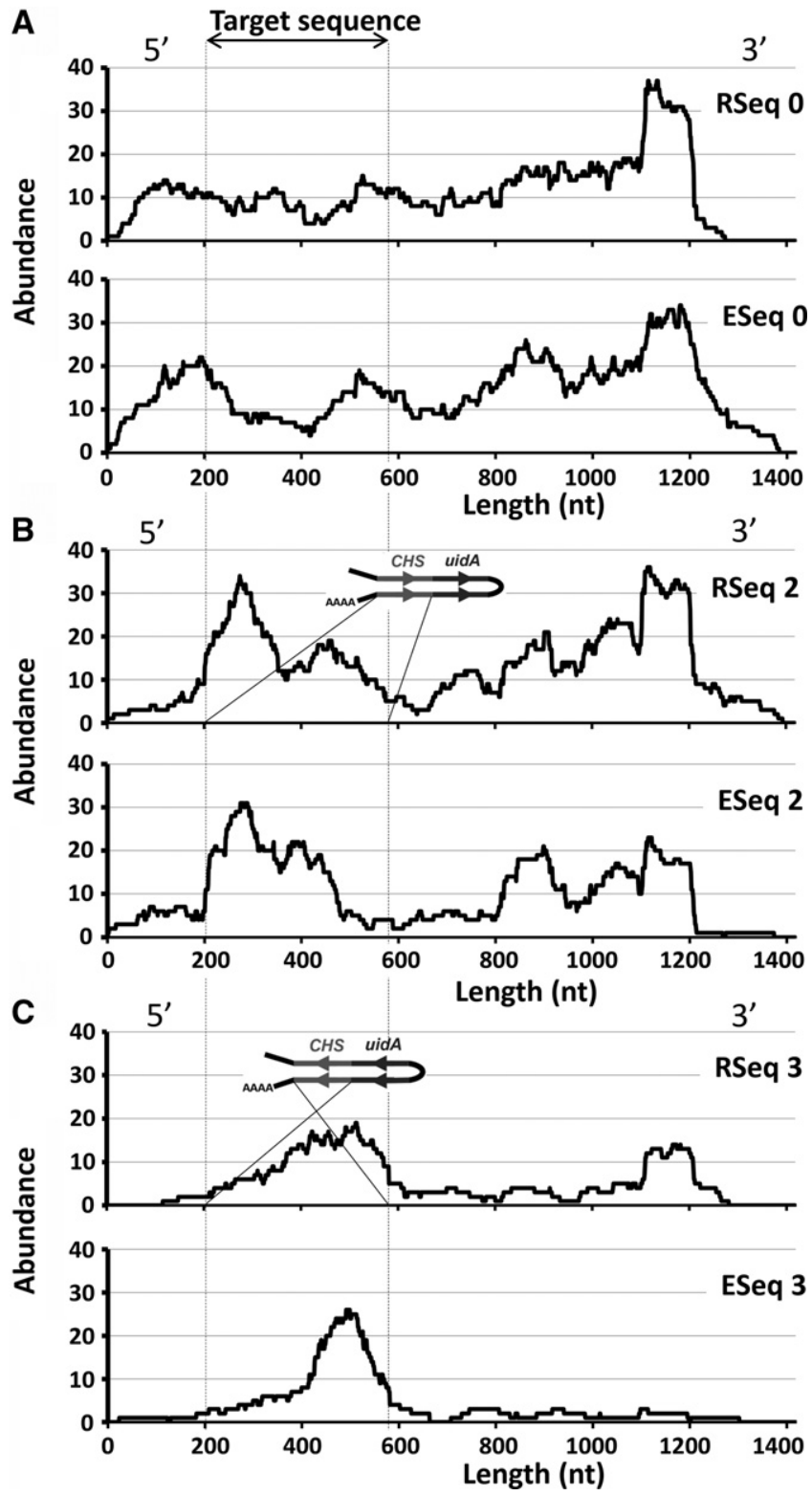

FIGURE 4. Alignment of reads from RSeq and ESeq libraries to the sequence of AtCHS mRNA. (A) In wild-type plants the reads were distributed across the entire length of AtCHS mRNA sequence; treatment with $5^{\prime} \mathrm{PDE}$ (ESeq 0 ) resulted in a slight increase in the coverage. (B) In RSeq 2, made from the plants transformed with chs $^{\mathrm{S}}$-gus ${ }^{\mathrm{S}} \wedge$ gus $^{\mathrm{A}}-$-chs $^{\mathrm{A}}$ and least efficient in silencing of AtCHS mRNA, the read coverage upstream $\left(5^{\prime}\right)$ of the target sequence is clearly reduced, but the coverage downstream $\left(3^{\prime}\right)$ is comparable to that in wild-type plants. The reads corresponding to the $3^{\prime}$ fragment originated from RNA sensitive to $5^{\prime} \mathrm{PDE}$ treatment, as indicated by reduced coverage in ESeq 2. (C) Even greater reduction in AtCHS mRNA-derived reads was observed among plants transformed with chs $^{\mathrm{A}}$-gus ${ }^{\mathrm{A}} \wedge$ gus $^{\mathrm{S}}$-chs ${ }^{\mathrm{S}}$. In both $(B)$ and $(C)$ the transgene-derived reads are more abundant toward the predicted poly-A tail within the ihpRNA structure. Coverage indicates number of corresponding reads per nucleotide out of 9 million total reads per library.

the same processes. In contrast, the $5^{\prime}$ fragment of the cleavage reaction is likely to be degraded by the exosome (Parker and Song 2004); accordingly, we did not find any evidence for 
primer-dependent $5^{\prime}$ spreading of sRNA production that would be indicative of $5^{\prime}$ transitivity (Fig. 3C; Sijen et al. 2001; Voinnet 2008).

\section{Position of peaks of sRNA accumulation may be determined by rare codons}

Our data indicated that sRNA production was primarily associated with the presence of ORFs. Therefore, we explored characteristics of the trigger sequence that might correlate with the observed peaks of sRNA accumulation. Previous reports (De Paoli et al. 2009; Kasai et al. 2013) suggested that secondary structures in CHALCONE SYNTHASE A (CHS-A) mRNA may trigger production of both endogenous and transgene-derived sRNAs during sense-mediated PTGS (S-PTGS) in petunia (Napoli et al. 1990). Consistent with this proposal, one of the five major peaks (P2) (Fig. 3A) in our At-CHS trigger sequence corresponded to an extended area of predicted self-complementarity in At-CHS mRNA that additionally resembles pre-microRNA (Supplemental Fig. S3). However, because our analyses showed that the biogenesis of sRNAs was associated with the presence of ORFs, we also examined whether the presence of rarely utilized codons coincided with sRNA accumulation. We adapted a previously published algorithm (Clarke and Clark 2008) to calculate the relative codon frequency for each amino acid in Arabidopsis based on codon usage (http://www.kazusa.or. $\mathrm{jp} / \mathrm{codon} /$ ) and assigned a rarity value to each codon.

For the At-CHS fragment used in our ihpRNA constructs we did not find a correlation between sRNA accumulation and codon frequency; however, the regions in the ihpRNA uidA fragment corresponding to peaks of sRNA were weakly but significantly $(P \leq 0.01)$ correlated with elevated frequencies of rare codons (Supplemental Fig. S4). We performed similar analyses on the $C H S-A$ gene from petunia using relative codon frequencies calculated for this species. Most of the CHS-A ORF comprises frequently used codons in petunia; however, a small central region contains a substantially elevated abundance of rare codons (Fig. 5). This region coincides precisely with the most abundant transgene-derived sRNAs in petals of cosuppressed petunia plants (Fig. 5). Because the full-length $C H S-A$ mRNA had no strong detectable sequence complementarities near the major peak for sRNA accumulation (data not shown), the peak's position appears more consistent with the elevated frequency of rare codons than with complementarity in the predicted mRNA secondary structure as suggested previously (De Paoli et al. 2009; Kasai et al. 2013).

\section{IR-PTGS affects the prevalence of endogenously produced sRNAs}

Processing of highly abundant, transgene-derived ihpRNAs engages the molecular components normally involved in biogenesis of endogenous sRNA. To evaluate the effect of

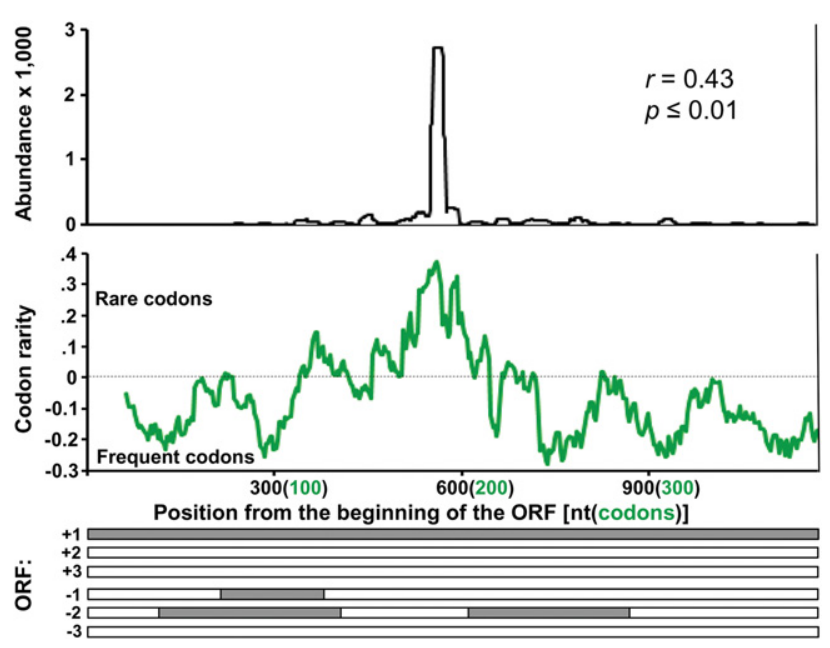

FIGURE 5. Distribution of sRNA associated with S-PTGS in petunia. Abundance of sRNAs mapped to the petunia CHS-A sequence (De Paoli et al. 2009) (top panel) and the frequency of rare codons in the sequence (middle panel) showing that the peak of sRNA production is correlated with a high abundance of rare codons and is independent of predicted ORFs (bottom panel). $(r)$ correlation coefficient.

IR-PTGS on endogenous sRNA accumulation, we analyzed the size distribution of sRNAs in four libraries made from moderately silenced plants (Libs 1-4), two libraries made from strongly silenced plants (Libs 5 and 6) (Fig. 1), and the reference library made from wild-type plants (Lib 0 ). In Lib 0 , the number of 20- to 21-nt long species approached 401,000 sequences per million (tspm) compared with 488 tspm of the 23- to 24-nt long species (Fig. 6). Among moderately silenced plants (Libs 1-4), the average abundance of 20- to 21-nt long sRNA species was still lower than the 23- to 24-nt long species at 419 and 445 tspm, respectively. However, in both libraries obtained from strongly silenced plants (Libs 5 and 6), the number of 20- to 21 -nt long reads increased to 541 and 602 tspm (1.4- and 1.5-fold increases, respectively) and the number of 23- to 24-nt long reads decreased to 322 and 269 tspm (1.5- and 1.8-fold decreases, respectively) (Fig. 6). The difference could not be attributed to the additional 20- to 21-nt long transgene-derived sRNAs because their abundance did not exceed $10 \%$ of the total number of sRNAs in any library analyzed.

To identify particular sRNA species responsible for observed differences, we mapped the sRNAs from three libraries (Libs 0, 5, and 6) to the five Arabidopsis chromosomes. Next, assuming that sRNA causing the size shift may represent only a fraction of the size class, we searched for loci for which the levels of corresponding sRNA differed by $>1.5$-fold between strongly silenced plants (Libs 5 and 6 ) and the control plants (Lib 7). Loci were defined as fragments of the reference sequence in which at least 10 sequential nucleotides were represented by at least 10 sequences per million in each of the three libraries analyzed. Differences were considered significant when they were detected in both the comparisons of Lib 5-Lib 0 and Lib 6-Lib 0. We identified 141 loci for 


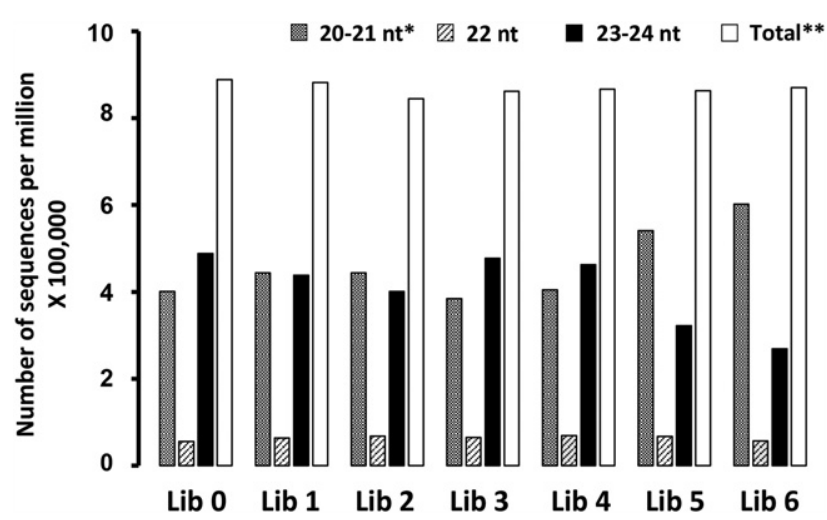

FIGURE 6. Distribution of different size classes of endogenous sRNA in the control library (Lib 0 ), four libraries made from moderately silenced plants (Libs 1-4), and two libraries made from strongly silenced plants (Libs 5 and 6). A clear increase in 20- to 21-nt long species and a decrease in 23- to 24-nt long species occur in strongly silenced plants. (*) All 20- to 24-nt long sequences matching the sequence of five Arabidopsis chromosomes. ${ }^{(*)}$ ) 20-nt long sRNA accounted for roughly one-third to half of the 20- to 21-nt size class.

which the corresponding 20- to 21-nt long sRNAs were overrepresented in strongly silenced plants (Supplemental Fig. S5). These comprised 22 loci encoding known miRNAs, including MIR156b, and five loci encoding tasiRNAs, one of which was TAS2 (Peragine et al. 2004; Vazquez et al. 2004; Allen et al. 2005; Jones-Rhoades et al. 2006). We also identified 43 loci in which the corresponding 20 - to 21-nt long sRNAs were under-represented in strongly silenced plants (Supplemental Fig. S6); among these were four loci that encode transcripts known to be the targets of TAS2 and MIR156b. This provides biological support for the observed increases in the abundance of these two regulatory sRNAs (Supplemental Fig. S5). Among the loci responsible for the production of 23- to 24-nt long species, we found 36 for which the number of corresponding sRNAs was increased. Most importantly, however, 3086 loci were found for which corresponding sRNAs were decreased in strongly silenced plants. Mapping of these sRNAs to the sequences of the five Arabidopsis chromosomes (Fig. 7) showed their prevalence in pericentromeric regions, characteristic of hc-siRNA (Lu et al. 2006). Abundance of sRNAs corresponding to 2131 of these 3086 loci decreased by more than twofold, which is more than the overall 1.5 - to 1.8 -fold decrease in 23- to 24-nt long sRNA species we observed in strongly silenced plants. Because hc-siRNAs contribute substantially to the overall prevalence of the 23to 24-nt long sRNA species in Arabidopsis, we concluded that strong IR-PTGS affects the prevalence of endog- enous sRNAs, primarily by decreasing accumulation of 23- to 24-nt long hc-siRNAs.

\section{DISCUSSION}

IR-PTGS is a commonly used approach to experimentally down-regulate gene expression in plants. Contemporary guidelines for designing constructs to maximize the efficiency of silencing have often been inferred from isolated successful cases and/or limited numbers of transgenic events. Here we provide extensive evaluation of four different constructs specifically designed to investigate the efficacy of RNAi induction based on 239 independent $T_{1}$ transgenic plants, several thousand transgenic progenies, and detailed analyses of the different RNA species associated with silencing. We conclude that to maximize the efficiency of IR-PTGS, the first (5'-most) arm of the IR should contain the trigger sequences in an antisense orientation. The sequences closer to the intron separating the arms of the IR produce more sRNAs and therefore trigger PTGS more efficiently (Fig. 1).

In our experiments, the majority of the transgene-derived sRNAs were produced from the 500- to 600-nt long region adjacent to the loop of the ihpRNA, and the relationship of sRNA abundance to distance from the loop (Fig. 2) implies that the use of trigger sequences totaling $>800 \mathrm{nt}$ is undesirable. Accordingly, if multiple genes are to be silenced using a single chimeric ihpRNA, the length of each sequence should be adjusted proportionally depending on its position in the ihpRNA. The enhanced sRNA production from fragments of ihpRNA adjacent to the predicted loop (Fig. 2) may reflect the dynamics of dsRNA assembly. The two complementary RNA strands may achieve physical proximity more quickly at the splicing junction, resulting in formation of the dsRNA that subsequently triggers production of abundant sRNAs.

In earlier reports we implemented silencing of transiently expressed GUS as a reporter for silencing used in multiple
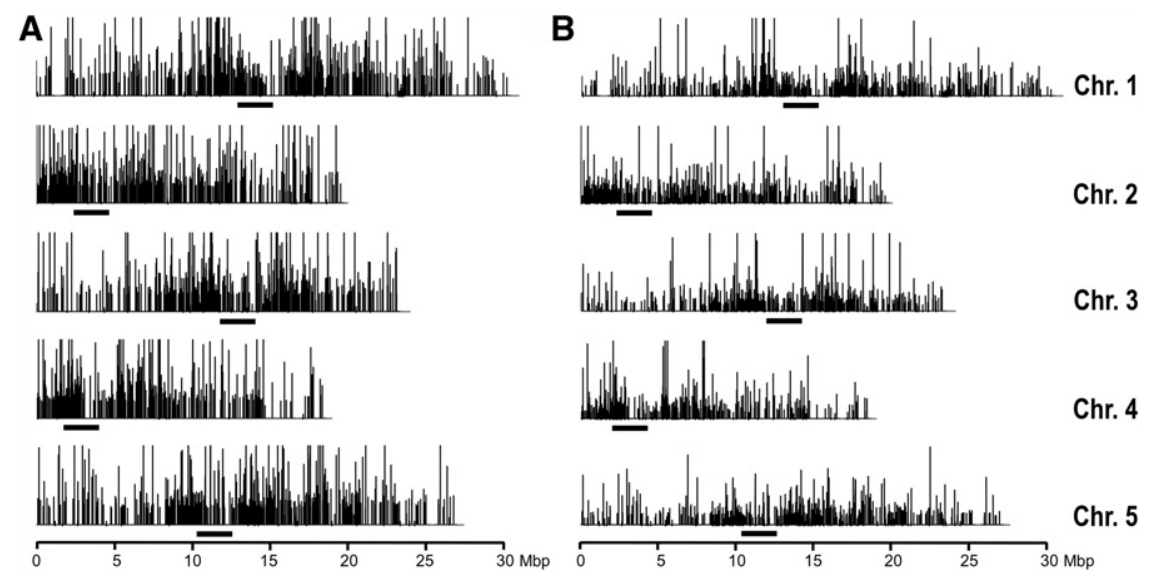

FIGURE 7. Chromosomal distribution of 23- to 24-nt long sRNAs in Arabidopsis plants. (A) Wild-type control plants (Lib 0) and (B) strongly silenced plants (Lib 6). Bars indicate the approximate positions of the centromeres. 
generations of transgenic lettuce plants by incorporating a fragment of the uidA gene into the chimeric ihpRNA construct (Wroblewski et al. 2007). Using information presented in this manuscript, the system was further optimized by moving the fragment of the uidA gene to the $5^{\prime}$ end of the ihpRNA and reducing its length from 342 to $300 \mathrm{bp}$. This made silencing of GUS less effective but also more indicative and reliable as a reporter for simultaneous IR-PTGS of the targeted gene. This improved configuration of the ihpRNA construct enabled us to successfully use reverse genetics to dissect complex disease resistance loci and demonstrate host-induced gene silencing as a strategy for resistance to biotrophic pathogens in lettuce (M Christopoulou and M Govindarajulu, unpubl.).

Several previously reported observations place the RNAi process in the nuclear compartment of the plant cell. DCL4, which is primarily involved in the processing of perfectly complementary dsRNAs such as those comprising ihpRNA, resides exclusively in the nucleus (Kumakura et al. 2009; Hoffer et al. 2011; Jouannet et al. 2012). In addition, intron sequences alone have been sufficient to trigger IR-PTGS, suggesting that not only ihpRNA processing but the entire process of IR-PTGS can be carried out in the nucleus as well (Hoffer et al. 2011). Consistent with nuclear positioning of the RNAi machinery, extensive IR-PTGS in our transgenic plants considerably altered levels of endogenous sRNA produced in the nucleus. Nucleoli are considered to be the processing centers for both DCL3-dependent hc-siRNAs and DCL1-dependent miRNAs (Pontes and Pikaard 2008), levels of which showed significant changes in our strongly silenced plants (Fig. 7; Supplemental Figs S5, S6).

The high levels of dsRNA produced during IR-PTGS have been suggested to promote the activities of DCLs that would normally act in other RNA silencing pathways (Brodersen and Voinnet 2006). DCL4 and DCL2, for example, are both capable of processing long, perfectly complementary dsRNA (Brodersen and Voinnet 2006). DCLs are associated with the production of particular sRNA size classes (Brodersen and Voinnet 2006) and DCL3 is normally involved in the biogenesis of hc-siRNAs that are 24-nt long. Therefore, DCL3 is unlikely to have been recruited to process ihpRNA in our experiments since ihpRNA-derived sRNAs are primarily 20 - to 21-nt long. Instead, the increase of more than two dozen specific miRNAs and ta-siRNA species (Supplemental Fig. S5) and the profound decrease of 23- to 24-nt long hc-siRNA species (Fig. 6) in our strongly silenced Arabidopsis plants resemble changes in endogenous sRNA production observed in the absence of RDR2 (Lu et al. 2006). This would imply, at least in the strongly silenced plants, the involvement of RDR2 or another molecular component of the RDR2-dependent sRNA pathway in ihpRNA processing. Interestingly, RDR2, as well as other components of the hc-siRNA pathway such as CLSY1 and NRPD1a, has previously been linked to short-distance signaling during IR-PTGS (Dunoyer et al. 2005, 2007; Smith et al. 2007; Brosnan and Voinnet 2011).
Another interesting observation in our experiments is that sequence translatability has a negative quantitative effect on sRNA production from ihpRNA, which is contradictory to the notion of IR-PTGS occurring in the nucleus since canonical translation takes place in the cytoplasm. The overall decrease in sRNA accumulation associated with the presence of ORFs, particularly from the $5^{\prime}$ fragment of ihpRNA (Fig. 1 ), suggests some level of translation and competition between the translation and RNAi machineries. A competition for single-stranded mRNA templates during RNA-mediated uncapped mRNA decay has been previously proposed (Gazzani et al. 2004; Gregory et al. 2009) and it was attributed to the limited access of RDR to the mRNA being translated and its inability to synthesize the complementary strand. In theory, because DCL-mediated processing of ihpRNA should not require synthesis of the complementary strand, the initiation of translation and association of ihpRNA with ribosomes may simply prevent its folding to form the doublestranded template for DCL. However, the observations discussed below suggest that processing of ihpRNA to produce sRNA may be more complex than simple DCL4- or DCL2mediated cleavage of dsRNA.

Mapping of ihpRNA-derived sRNAs in our transgenic Ws0 plants to their corresponding sequences revealed clearly delineated peaks (Fig. 3). The positions of these peaks cannot be explained by sequential DCL4-mediated cleavage because they were not dependent upon ihpRNA configuration. Although both fragments were linked together in one ihpRNA molecule, the positions of the peaks corresponding to the At-CHS fragment were consistent and completely independent of the positions of the peaks corresponding to the uidA fragment. In addition, the five major peaks corresponding to the At-CHS gene fragment were distributed in approximate 79-nt intervals (Fig. 3A,B), which does not fit with the 21-nt phasing observed after sequential DCL4-mediated cleavage (Napoli et al. 1990; Howell et al. 2007; De Paoli et al. 2009; Kasai et al. 2013). In fact, the positions of the peaks corresponding to the At-CHS fragment used to generate ihpRNA were the same as those of the peaks produced by sRNAs associated with uncapped RNA decay of At-CHS mRNA in the ein5/abh1-1 mutant (Gregory et al. 2009). This suggests that the determinant(s) of the positions of sRNA peaks in uncapped mRNA decay and ihpRNA processing are likely the same.

To investigate the determinants of the positions of sRNA peaks observed during IR-PTGS, RNAi-mediated ssRNA decay and S-PTGS, we evaluated several factors. We investigated the $5^{\prime}$ terminal nucleotides that may affect the ability of sRNA to bind AGO (Mi et al. 2008) but we were not able to find a clear preference for any particular nucleotide (data not shown). This was consistent with peaks being composed of overlapping and diverse sRNA species. Examination of the predicted secondary structure of At-CHS mRNA revealed that only one of the five major peaks in the trigger sequence corresponded to an extended length of self-complementarity 
(Supplemental Fig. S3). The positions of other peaks did not match predicted double-stranded fragments of At- $\mathrm{CHS}$ mRNA. In contrast, in multiple cases we detected associations between the distribution of peaks and the presence of primary or secondary ORFs (Fig. 3B,C; Supplemental Fig. S2). These data indicate that although presence of an ORF can decrease overall sRNA accumulation (Fig. 1), it can also determine the positions of sRNA peaks corresponding to the trigger sequence.

In addition to the quantitative effects discussed above, sequence translatability also has qualitative effects on sRNA production. The position of peaks of sRNA accumulation correlated with the position of predicted ORFs (Fig. 3; Supplemental Fig. S2). Furthermore, translatability can be influenced by several factors such as mRNA secondary structure and codon usage (Fig. 5; Supplemental Figs. S3, S4), both of which may cause ribosome stalling (Shoemaker and Green 2012). The 79-nt phasing of the peaks of sRNA produced from At-CHS (Fig. 3A,B) may therefore correspond to the positions of stalled ribosomes and/or the spaces in between stalled ribosomes that are accessible to the RNAi machinery. The facilitating role for SDE3 helicase during RDR6-mediated amplification of sRNA (Dalmay et al. 2000; Mourrain et al. 2000; Curaba and Chen 2008; Garcia et al. 2012) suggests a speculative but attractive explanation for the correlation between sRNA distribution and the presence of ORFs (Fig. 3; Supplemental Fig. S2). RNAi-mediated RNA decay and transitivity both involve RDR-mediated synthesis of dsRNA from single-stranded (ss) RNA templates that are likely perceived in the cell as aberrant (De Paoli et al. 2009; Gregory et al. 2009). Perhaps preferential accumulation of sRNA corresponding to ORFs in such aberrant mRNA (Fig. 3; Supplemental Fig. S2) could occur as a result of amplification involving the helicase activity of ribosomes (Takyar et al. 2005) working in conjunction with RDR6. Previously suggested competition between the translational machinery and RDRs for mRNA substrates (Gazzani et al. 2004; Gregory et al. 2009) could provide a sensitive mechanism for detecting aberrant RNA, exposing such RNA to RDR6 particularly during ribosome stalling. Amplification of sRNA corresponding to that aberrant RNA template could then possibly occur by a mechanism similar to that proposed by Garcia et al. (2012). Furthermore, the initially produced sRNA acting as translational repressors (Brodersen et al. 2008) could, in turn, increase ribosome stalling, RDR6 recruitment, and subsequent biogenesis of secondary sRNA, thereby resulting in clearly delineated peaks of sRNA accumulation. Whether or not a combination of inefficient translation and the helicase activity of ribosomes contribute to ihpRNA processing is an open question. However, the weak but significant correlation we observed between the distribution of sRNA corresponding to the uidA (GUS) target sequence in our experiments and the occurrence of rare codons in that fragment (Supplemental Fig. S4) serves as additional, indirect support for such a possibility.
In conclusion, our extensive analyses of sRNA associated with IR-PTGS in Arabidopsis provide insights into the interplay of IR-PTGS and endogenous pathways of sRNA biogenesis, as well as provide guidelines for designing ihpRNA constructs to optimize their efficiency for inducing PTGS. The similarities in patterns of sRNA production suggest that similar mechanisms underlie ihpRNA processing and RNAi-mediated decay involved in mRNA turnover. Finally, the profound effect of IR-PTGS on hc-siRNA, miRNAs, and ta-siRNAs may result in off-target effects that should be taken into consideration when interpreting RNA silencing experiments as well as implementing RNAi strategies for agricultural purposes.

\section{MATERIALS AND METHODS}

Sequences from this article can be found in the GEO data libraries under accession number GSE38922 (http://www.ncbi.nlm.nih.gov/ geo/query/acc.cgi?acc=GSE38922).

\section{Production of transgenic plants and assays for gene silencing}

A 372-bp fragment of the At-CHS gene (Gene Bank \# 03, bases 359730 ) and a 342-bp fragment of the uidA gene (Gene Bank \# M14641, bases 2430-2771) were fused together in different configurations and cloned into a modified version of binary vector pGSA1165 (http://chromdb.org/), in sense and antisense orientations (Fig. 1; Supplemental Fig. S1). Two arms of the inverted repeat in each construct were separated by intron-3 of the PYRUVATE ORTHOPHOSPHATE DIKINASE gene from Flaveria trinervia (FtPDK; Gene Bank \# X79095, bases 7890-8668) (Supplemental Fig. S1). In the two constructs containing the fused gene fragments adjacent to the promoter in the sense orientation, the fused fragments were cloned in frame with the ATG codon downstream from the $35 \mathrm{~S}$ promoter in the vector backbone creating ORFs (Supplemental Fig. S1). (Details of vector modification and cloning are provided in Supplemental Materials and Methods.) All constructs were introduced into Agrobacterium tumefaciens strain LBA4404. Transgenic plants of Arabidopsis thaliana ecotype Wassilewskija (Ws-0; CS 915; TAIR, http://www.arabidopsis.org/) were produced using floral dip transformation (Clough and Bent 1998). Transient expression of GUS was induced in $\mathrm{T}_{1}$ plants using binary vector plasmid pTFS40 containing the uidA gene as described previously (Wroblewski et al. 2005). Six days after infiltration two to three leaves from each plant were pooled together for protein extraction and quantitative evaluation of GUS activity using 4-methylumbelliferyl- $\beta$-D-glucuronide (MUG)-based fluorimetrical assay (Jefferson et al. 1987) in microtiter plates with an Analyst $\mathrm{AD}$ instrument (Molecular Devices, http://www.moleculardevices.com/) at an excitation wavelength of $360 \mathrm{~nm}$ and an emission wavelength of $460 \mathrm{~nm}$. Induction of transient GUS expression and GUS activity measurement were performed three times in each $\mathrm{T}_{1}$ plant and in three nontransgenic Ws-0 plants as a control. Data presented in Figure 1B show average values obtained from these three replicates, were standardized to total protein content in the extracts, and comprise over 700 measurements. The same values were also used for correlation analysis. Silencing of At-CHS was visually evaluated based on the level of 
anthocyanin production in $\mathrm{T}_{2}$ plants. The $\mathrm{T}_{2}$ families were randomized in a growth chamber and grown in three replicas under stressful conditions: light intensity of $\sim 300 \mu \mathrm{E}, 14 \mathrm{~h}$ light/10 h dark photoperiod, $15^{\circ} \mathrm{C}$ during the day and $5^{\circ} \mathrm{C}$ during the night. After $4 \mathrm{wk}$, each plant was assigned to one of four phenotypic categories as shown in Figure 1C: red-anthocyanin levels similar to wild-type, no silencing; red-yellow-coloration strong but weaker than the wild-type, weak silencing; yellow-green-only traces of anthocyanin present in all leaves, strong silencing; and green-no anthocyanin detected, complete silencing. The efficacy of silencing in each $\mathrm{T}_{2}$ family was computed as the number of completely green plants plus half of all the plants with only traces of anthocyanin divided by the total number of plants analyzed for that family. At least 48 individual plants were evaluated per each $T_{2}$ family for a total of over 17,000 plants. (For more details on plant assays and data analyses see Supplemental Materials and Methods.)

\section{Construction of sRNA libraries and sequencing}

Each sRNA library was made from RNA isolated from 120 plants grown for 4 wk under stressful conditions as described above and pooled together. Ten silenced plants (lacking anthocyanin) from each of 12 selected $\mathrm{T}_{2}$ families per construct (indicated with a white rectangle in Fig. 1C) were harvested and used for total RNA extraction using Trizol (Invitrogen Inc.; http://www.invitrogen.com/). The libraries were made using Small RNA Sample Prep Kits (Illumina Inc.; http://www.illumina.com/) following the manufacturer's protocol. Gel-purified fractions of sRNA from $10 \mu \mathrm{g}$ total RNA were used as an input material. Small RNAs were ligated to Illumina-supplied $5^{\prime}$ and $3^{\prime}$ adapters. The products of amplification corresponding to 18 - to 30-nt long RNAs were excised from Novex 6\% TBE PAGE gel (Invitrogen Inc.; http://www.invitrogen.com/) and sequenced on the Genome Analyzer II using 41 (Libs 0, 5, and 6) or 45 (Libs 1-4) cycles.

\section{Analysis of sRNAs}

Sequences were extracted from the GAII image files using the standard Illumina pipeline (http://code.google.com/p/atgc-illumina/ source/browse/\#svn/trunk). Details related to sig2 base-calling are available at https://code.google.com/p/atgc-illumina/. A custom Perl script was used to identify and trim adapter sequences. Because no substantial differences were found between libraries after alignment to a set of 40 genomic reference loci, no further normalization was performed. Reads lacking adapter sequences or shorter than $18 \mathrm{nt}$ after trimming were removed from further analysis. Five hundred thousand sequences from each library were mapped to four query sequences corresponding to the four different constructs using BLAST (Altschul et al. 1990) with a word size of 10 and expectation value of 0.1. A custom Python script (http://code. google.com/p/xuhu-rwm-blast/wiki/Blastcovct) was used to generate tables with coverage values per nucleotide of query sequence. Significance of the position-dependent differences in sRNA production among the four constructs was determined using the $\chi^{2}$ test and the cumulative values of sRNA representations for each fragment, At-CHS or uidA. Correlation between abundance of sRNAs in peaks and their position in an ihpRNA (Fig. 2) was calculated using the distance from loop (nt) and sRNA abundance in ten peaks standardized to the mean. Analyses of endogenous sRNAs were performed using 1 million randomly selected sequences from each library and the reference sequence of five Arabidopsis chromosomes (http://www.ncbi.nlm.nih.gov). The alignments were created using CLC Genomics Workbench 4.9 (http://www.clcbio.com/) and the results, retrieved as SAM formatted files, were filtered using a custom Python script (http://code.google.com/p/xuhu-rwm-blast/ wiki/Blastcovct) that also calculated and compared the coverage of 20-21 and 23-24 sequences from each library over each of the five Arabidopsis chromosomes.

\section{Calculation of codon usage, prediction of ORFs, and determination of mRNA secondary structures}

For A. thaliana and Petunia hybrida, we used a codon usage table calculated for all coding sequences (http://www.kazusa.or.jp/). The relative codon frequency (RCF; rarity) was calculated using an approach similar to that published previously (Clarke and Clark 2008). For the $j$ th codon of the $i$ th amino acid with $\mathrm{n}$ synonymous codons, the algorithm calculates the RCF factor by which the actual codon usage frequency $\left(X_{i j}\right)$ differs from the average frequency of synonymous codons for a particular amino acid $\left(X_{\mathrm{avg}, i}\right)$ :

$$
\begin{aligned}
& X_{\mathrm{avg}, i}=\frac{1}{n_{i}} \sum_{j=1}^{n_{i}} X_{i j} \\
& \text { If } X_{i j}>X_{\mathrm{avg}, i} \text { then } \mathrm{RFC}=-\left(\frac{X_{i j}}{X_{\mathrm{avg}, i}}-1\right) \\
& \text { If } X_{i j}<X_{\mathrm{avg}, i} \text { then } \mathrm{RFC}=-\left(1-\frac{X_{i j}}{X_{\mathrm{avg}, i}}\right) \\
& \text { If } X_{i j}=X_{\mathrm{avg}, i} \text { then } \mathrm{RFC}=0
\end{aligned}
$$

For clarity of display we multiplied the RFC by negative 1 , and therefore the less frequent a particular codon among all synonymous codons, the higher the value assigned to it and vice versa. The relative abundance of rare codons presented in Figure 4 shows the average values for RCF calculated using for 20-codons long sliding (by one codon) windows. The $X_{i j}$ values were obtained from the Kazusa DB. Presence of ORFs was predicted using a publicly available online tool (http://www.ncbi.nlm.nih.gov/projects/gorf/). Correlations between codon frequency and sRNA production were analyzed using RFC value for each codon and the average abundance of sRNA over the three nucleotides comprising that codon using Microsoft Excel. Significance of these correlations was determined using critical $P$ values in the two-tailed test. Secondary mRNA structures were determined by Mfold (Zuker 2003) with default settings. Most calculations, statistical analyses and data displays were carried out using Microsoft Excel.

\section{Construction of polyA RNA libraries and analysis of RNA-Seq data}

RNA-Seq libraries were made using poly-A RNA purified from total RNA preparations obtained for sRNA libraries (described above) using Dynabeads oligo $(\mathrm{dT})_{25}$ and manufacturer's protocol (Dynabeads mRNA direct kit; http://www.lifetechnologies.com). The cDNA was synthesized and fragmented to obtain 250-bp insert size using Covaris (http://covarisinc.com) according to a previously published protocol (Zhong et al. 2011) except for PCR enrichment. Each library was barcoded using Illumina-compatible NEXTflex 
DNA Barcodex (http://www.biooscientific.com). Individual libraries were pooled and sequenced using Illumina HiSeq 2000 sequencer to obtain $100 \times 100 \mathrm{nt}$ paired-end reads. The raw Illumina sequence reads were processed for barcode removal (CASAVA v1.8), adapter removal (Scythe program), filtered and trimmed for quality (allPrep script by M. Lieberman, University of California Davis; http://comailab.genomecenter.ucdavis.edu/index.php/Barcoded _data_preparation_tools). Nine million randomly selected reads from each library were used for further analysis. Alignment to Arabidopsis rRNA-5S-encoding sequences, thirteen reference sequences used previously for transcript normalization (Czechowski et al. 2005), and to the At-CHS mRNA sequence was performed using CLC Genomics Workbench (http://www.clcbio.com).

\section{SUPPLEMENTAL MATERIAL}

Supplemental material is available for this article.

\section{ACKNOWLEDGMENTS}

We thank Anna Tomczak and Justin Warren for technical support, Charlie Nicolet from DNA Technologies Core at the Genome Center (UC Davis) for sequencing, and several UC Davis and external colleagues for discussion and critical reading of the manuscript. This work was supported by awards from the National Science Foundation Plant Genome and Arabidopsis 2010 programs (grant numbers 0822393 and 0820451).

Received May 22, 2014; accepted September 11, 2014.

\section{REFERENCES}

Allen E, Xie Z, Gustafson AM, Carrington JC. 2005. MicroRNA-directed phasing during trans-acting siRNA biogenesis in plants. Cell 121: 207-221.

Altschul SF, Gish W, Miller W, Myers EW, Lipman DJ. 1990. Basic local alignment search tool. J Mol Biol 215: 403-410.

Bartel DP. 2004. MicroRNAs: genomics, biogenesis, mechanism, and function. Cell 116: 281-297.

Bernstein E, Caudy AA, Hammond SM, Hannon GJ. 2001. Role for a bidentate ribonuclease in the initiation step of RNA interference. Nature 409: 363-366.

Bouche N, Lauressergues D, Gasciolli V, Vaucheret H. 2006. An antagonistic function for Arabidopsis DCL2 in development and a new function for DCL4 in generating viral siRNAs. $E M B O J$ 25: $3347-3356$.

Brodersen P, Voinnet O. 2006. The diversity of RNA silencing pathways in plants. Trends Genet 22: 268-280.

Brodersen P, Sakvarelidze-Achard L, Bruun-Rasmussen M, Dunoyer P, Yamamoto YY, Sieburth L, Voinnet O. 2008. Widespread translational inhibition by plant miRNAs and siRNAs. Science 320: $1185-1190$.

Brosnan CA, Voinnet O. 2011. Cell-to-cell and long-distance siRNA movement in plants: mechanisms and biological implications. Curr Opin Plant Biol 14: 580-587.

Brosnan CA, Mitter N, Christie M, Smith NA, Waterhouse PM, Carroll BJ. 2007. Nuclear gene silencing directs reception of longdistance mRNA silencing in Arabidopsis. Proc Natl Acad Sci 104: 14741-14746.

Chuang CF, Meyerowitz EM. 2000. Specific and heritable genetic interference by double-stranded RNA in Arabidopsis thaliana. Proc Nat Acad Sci 97: 4985-4990.

Clarke TF IV, Clark PL. 2008. Rare codons cluster. PLoS One 3: e3412.
Clough SJ, Bent AF. 1998. Floral dip: a simplified method for Agrobacterium-mediated transformation of Arabidopsis thaliana. Plant J 16: 735-743.

Curaba J, Chen X. 2008. Biochemical activities of Arabidopsis RNAdependent RNA polymerase 6. J Biol Chem 283: 3059-3066.

Czechowski T, Stitt M, Altmann T, Udvardi MK, Scheible W-R. 2005. Genome-wide identification and testing of superior reference genes for transcript normalization in Arabidopsis. Plant Physiol 139: 5-17.

Dalmay T, Hamilton A, Rudd S, Angell S, Baulcombe DC. 2000. An RNA-dependent RNA polymerase gene in Arabidopsis is required for posttranscriptional gene silencing mediated by a transgene but not by a virus. Cell 101: 543-553.

Dalmay TD, Horsefield R, Braunstein TH, Baulcombe DC. 2001. SDE3 encodes an RNA helicase required for posttranscriptional gene silencing in Arabidopsis. EMBO J 20: 2069-2078.

De Paoli E, Dorantes-Acosta A, Zhai J, Accerbi M, Jeong DH, Park S, Meyers BC, Jorgensen RA, Green PJ. 2009. Distinct extremely abundant siRNAs associated with cosuppression in petunia. RNA 15: 1965-1970.

Denli A, Hannon G. 2003. RNAi: an ever-growing puzzle. Trends Biochem Sci 28: 196-201.

Dunoyer P, Voinnet O. 2008. Mixing and matching: the essence of plant systemic silencing? Trends Genet 24: 151-154.

Dunoyer P, Himber C, Voinnet O. 2005. DICER-LIKE 4 is required for RNA interference and produces the 21-nucleotide small interfering RNA component of the plant cell-to-cell silencing signal. Nat Genet 37: 1356-1360.

Dunoyer P, Himber C, Ruiz-Ferrer V, Alioua A, Voinnet O. 2007. Intraand intercellular RNA interference in Arabidopsis thaliana requires components of the microRNA and heterochromatic silencing pathways. Nat Genet 39: 848-856.

Garcia D, Garcia S, Pontier D, Marchais A, Renou JP, Lagrange T, Voinnet O. 2012. Ago hook and RNA helicase motifs underpin dual roles for SDE3 in antiviral defense and silencing of nonconserved intergenic regions. Mol Cell 48: 109-120.

Gasciolli V, Mallory AC, Bartel DP, Vaucheret H. 2005. Partially redundant functions of Arabidopsis DICER-like enzymes and a role for DCL4 in producing trans-acting siRNAs. Curr Biol 15: 1494-1500.

Gazzani S, Lawrenson T, Woodward C, Headon D, Sablowski R. 2004. A link between mRNA turnover and RNA interference in Arabidopsis. Science 306: 1046-1048.

Gregory BD, O’Malley RC, Lister R, Urich MA, Tonti-Filippini J, Chen H, Millar AH, Ecker JR. 2009. A link between RNA metabolism and silencing affecting Arabidopsis development. Dev Cell 14: 854-866.

Hamilton AJ, Baulcombe DC. 1999. A species of small antisense RNA in posttranscriptional gene silencing in plants. Science 286: 950-952.

Hamilton A, Voinnet O, Chappell L, Baulcombe DC. 2002. Two classes of short interfering RNA in RNA silencing. EMBO J 21: 4671-4679.

Hammond SM, Bernstein E, Beach D, Hannon GJ. 2000. An RNA-directed nuclease mediates post-transcriptional gene silencing in Drosophila cells. Nature 404: 293-296.

Hannon GJ. 2002. RNA interference. Nature 418: 244-251.

Henderson IR, Zhang X, Lu C, Johnson L, Meyers BC, Green PJ, Jacobsen SE. 2006. Dissecting Arabidopsis thaliana DICER function in small RNA processing, gene silencing and DNA methylation patterning. Nat Genet 38: 721-725.

Hoffer P, Ivashuta S, Pontes O, Vitins A, Pikaard C, Mroczka A, Wagner N, Voelker T. 2011. Posttranscriptional gene silencing in nuclei. Proc Natl Acad Sci 108: 409-414.

Howell MD, Fahlgren N, Chapman EJ, Cumbie JS, Sullivan CM, Givan SA, Kasschau KD, Carrington JC. 2007. Genome-wide analysis of the RNA-DEPENDENT RNA POLYMERASE6/ DICER-LIKE4 pathway in Arabidopsis reveals dependency on miRNA- and tasiRNA-directed targeting. Plant Cell 19: 926-942.

Jauvion V, Elmayan T, Vaucheret H. 2010. The conserved RNA trafficking proteins HPR1 and TEX1 are involved in the production of endogenous and exogenous small interfering RNA in Arabidopsis. Plant Cell 22: 2697-2709. 
Jefferson RA, Kavanagh TA, Bevan M. 1987. GUS fusions: $\beta$-glucuronidase as a sensitive and versatile gene fusion marker in higher plants. EMBO J 6: 3901-3907.

Jones-Rhoades MW, Bartel DP, Bartel B. 2006. MicroRNAs and their regulatory roles in plants. Annu Rev Plant Biol 57: 19-53.

Jouannet V, Moreno AB, Elmayan T, Vaucheret H, Crespi MD, Maizel A. 2012. Cytoplasmic Arabidopsis AGO7 accumulates in membrane-associated siRNA bodies and is required for ta-siRNA biogenesis. EMBO J 31: 1704-1713.

Kasai M, Matsumura H, Yoshida K, Terauchi R, Taneda A, Kanazawa A. 2013. Deep sequencing uncovers commonality in small RNA profiles between transgene-induced and naturally occurring RNA silencing of chalcone synthase-A gene in petunia. BMC Genomics 14: 63.

Kumakura N, Takeda A, Fujioka Y, Motose H, Takano R, Watanabe Y. 2009. SGS3 and RDR6 interact and colocalize in cytoplasmic SGS3/ RDR6-bodies. FEBS Lett 583: 1261-1266.

Law JA, Jacobsen SE. 2010. Establishing, maintaining and modifying DNA methylation patterns in plants and animals. Nat Rev Genet 11: 204-220.

Liu J, He Y, Amasino R, Chen X. 2004. siRNA s targeting an intronic transposon in the regulation of natural flowering behavior in Arabidopsis. Genes Dev 18: 2873-2878.

Llave C, Kasschau KD, Rector MA, Carrington JC. 2002. Endogenous and silencing-associated small RNAs in plants. Plant Cell 14: $1605-1619$.

Lu C, Kulkarni K, Souret FF, Muthuvalliappan R, Tej SS, Poethig RS, Henderson IR, Jacobsen SE, Wang W, Green PJ, et al. 2006. MicroRNAs and other small RNAs enriched in the Arabidopsis RNA-dependent RNA polymerase 2 mutant. Genome Res 16: 1276-1288.

Mi S, Cai T, Hu Y, Chen Y, Hodges E, Ni F, Wu L, Li S, Zhou H, Long C, et al. 2008. Sorting of small RNAs into Arabidopsis argonaute complexes is directed by the $5^{\prime}$ terminal nucleotide. Cell 133: 116-127.

Moissiard G, Parizotto EA, Himber C, Voinnet O. 2007. Transitivity in Arabidopsis can be primed, requires the redundant action of the antiviral Dicer-like 4 and Dicer-like 2, and is compromised by viral-encoded suppressor proteins. RNA 13: 1268-1278.

Mourrain P, Béclin C, Elmayan T, Feuerbach F, Godon C, Morel JB, Jouette D, Lacombe AM, Nikic S, Picault N, et al. 2000. Arabidopsis SGS2 and SGS3 genes are required for posttranscriptional gene silencing and natural virus resistance. Cell 101: 533-542.

Napoli C, Lemieux C, Jorgensen R. 1990. Introduction of a chimeric chalcone synthase gene into petunia results in reversible co-suppression of homologous genes in trans. Plant Cell 2: 279-289.

Parker R, Song H. 2004. The enzymes and control of eukaryotic mRNA turnover. Nat Struct Mol Biol 11: 121-127.

Peragine A, Yoshikawa M, Wu G, Albrecht HL, Poethig RS. 2004. SGS3 and SGS2/SDE1/RDR6 are required for juvenile development and the production of trans-acting siRNAs in Arabidopsis. Genes Dev 18: $2368-2379$.

Pontes O, Pikaard CS. 2008. siRNA and miRNA processing: new functions for Cajal bodies. Curr Opin Genet Dev 18: 197-203.

Pontier D, Picart C, Roudier F, Garcia D, Lahmy S, Azevedo J, Alart E, Laudie M, Karlowski WM, Cooke R, et al. 2012. NERD, a plant-specific GW protein, defines an additional RNAi-dependent chromatinbased pathway in Arabidopsis. Mol Cell 48: 121-132.

Shoemaker CJ, Green R. 2012. Translation drives mRNA quality control. Nat Struct Mol Biol 19: 594-601.
Sijen T, Fleenor J, Simmer F, Thijssen KL, Parrish S, Timmons L, Plasterk RHA, Fire A. 2001. On the role of RNA amplification in dsRNA-triggered gene silencing. Cell 107: 465-476.

Smith NA, Singh SP, Wang M-B, Stoutjesdijk PA, Green AG, Waterhouse PM. 2000. Gene expression: total silencing by intronspliced hairpin RNAs. Nature 407: 319-320.

Smith LM, Pontes O, Searle I, Yelina N, Yousafzai FK, Herr AJ, Pikaard CS, Baulcombe DC. 2007. An SNF2 protein associated with nuclear RNA silencing and the spread of a silencing signal between cells in Arabidopsis. Plant Cell 19: 1507-1521.

Souret FF, Kastenmayer JP, Green PJ. 2004. AtXRN4 degrades mRNA in Arabidopsis and its substrates include selected miRNA targets. Mol Cell 15: 173-183.

Takyar S, Hickerson RP, Noller HF. 2005. mRNA helicase activity of the ribosome. Cell 120: 49-58.

Tang G, Reinhart BJ, Bartel DP, Zamore PD. 2003. A biochemical framework for RNA silencing in plants. Genes Dev 17: 49-63.

Vaistij FE, Jones L, Baulcombe DC. 2002. Spreading of RNA targeting and DNA methylation in RNA silencing requires transcription of the target gene and a putative RNA-dependent RNA polymerase. Plant Cell 14: 857-867.

Vazquez F, Vaucheret H, Rajagopalan R, Lepers C, Gasciolli V, Mallory AC, Hilbert JL, Bartel DP, Crété P. 2004. Endogenous trans-acting siRNAs regulate the accumulation of Arabidopsis mRNAs. Mol Cell 16: 69-79.

Voinnet O. 2008. Use, tolerance and avoidance of amplified RNA silencing by plants. Trends Plant Sci 13: 317-328.

Voinnet O, Vain P, Angell S, Baulcombe DC. 1998. Systemic spread of sequence-specific transgene RNA degradation in plants is initiated by localized introduction of ectopic promoterless DNA. Cell 95: 177-187.

Wroblewski T, Tomczak A, Michelmore R. 2005. Optimization of Agrobacterium-mediated transient assays of gene expression in lettuce, tomato and Arabidopsis. Plant Biotechnol J 3: 259-273.

Wroblewski T, Piskurewicz U, Tomczak A, Ochoa O, Michelmore RW. 2007. Silencing of the major family of NBS-LRR-encoding genes in lettuce results in the loss of multiple resistance specificities. Plant $J$ 51: 803-818.

Xie Z, Johansen LK, Gustafson AM, Kasschau KD, Lellis AD, Zilberman D, Jacobsen SE, Carrington JC. 2004. Genetic and functional diversification of small RNA pathways in plants. PLoS Biol 2: 642-652.

Xie Z, Allen E, Wilken A, Carrington JC. 2005. DICER-LIKE 4 functions in trans-acting small interfering RNA biogenesis and vegetative phase change in Arabidopsis thaliana. Proc Natl Acad Sci 102: 12984-12989.

Zamore PD, Tuschl T, Sharp PA, Bartel DP. 2000. RNAi: Doublestranded RNA directs the ATP-dependent cleavage of mRNA at 21 to 23 nucleotide intervals. Cell 101: 25-33.

Zhong S, Joung JG, Zheng Y, Chen YR, Liu B, Shao Y, Xiang JZ, Fei Z, Giovannoni JJ. 2011. High-throughput illumina strand-specific RNA sequencing library preparation. Cold Spring Harb Protoc 2011: 940-949.

Zilberman D, Cao X, Jacobsen SE. 2003. ARGONAUTE4 control of locus-specific siRNA accumulation and DNA and histone methylation. Science 299: 716-719.

Zuker M. 2003. Mfold web server for nucleic acid folding and hybridization prediction. Nucleic Acids Res 31: 3406-3415. 

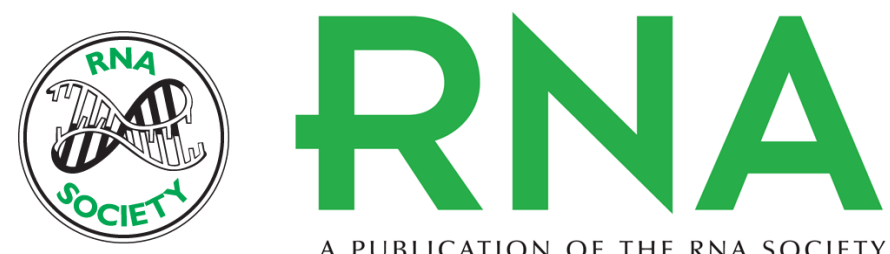

A PUBLICATION OF THE RNA SOCIETY

\section{Distinctive profiles of small RNA couple inverted repeat-induced post-transcriptional gene silencing with endogenous RNA silencing pathways in Arabidopsis}

Tadeusz Wroblewski, Marta Matvienko, Urszula Piskurewicz, et al.

RNA 2014 20: 1987-1999 originally published online October 24, 2014 Access the most recent version at doi:10.1261/rna.046532.114

Supplemental Material

References

Open Access

Creative Commons License

Email Alerting Service
http://rnajournal.cshlp.org/content/suppl/2014/10/08/rna.046532.114.DC1

This article cites 69 articles, 26 of which can be accessed free at: http://rnajournal.cshlp.org/content/20/12/1987.full.html\#ref-list-1

Freely available online through the RNA Open Access option.

This article, published in RNA, is available under a Creative Commons License (Attribution 4.0 International), as described at http://creativecommons.org/licenses/by/4.0/.

Receive free email alerts when new articles cite this article - sign up in the box at the top right corner of the article or click here. 